\title{
How apparent motion affects mental rotation: Push or pull?
}

\author{
PAUL M. CORBALLIS \\ Columbia University, New York, New York \\ and \\ MICHAEL C. CORBALLIS \\ University of Auckland, Auckland, New Zealand
}

\begin{abstract}
Subjects were timed as they judged whether a small bar perpendicular to one side of a clockhand would point left or right if the hand was pointing upward (i.e., at the 12:00 position). The clockhand was shown in two successive orientations $30^{\circ}$ apart, so that it was perceived to jump from one to the other, but the bar was included at only one of the two orientations. Analysis of reaction times as a function of angular orientation showed that the subjects "mentally rotated" the clockhand to the upright position before making their decisions. When the bar appeared on the second presentation, the jump had no significant influence on mental rotation but when it appeared on the first presentation, the estimated orientation from which the clockhand was mentally rotated was "dragged" in the direction of the jump.
\end{abstract}

Mental rotation is the act of imagining a shape or object turning around. Its properties have been inferred largely through the use of reaction time (RT) techniques. For example, Shepard and Metzler (1971) found that RT to judge whether two shapes depicted in different orientations were the same increased linearly with the angle between them, and inferred that their subjects mentally rotated one into congruence with the other in order to make the judgment. In this respect, mental rotation mimics physical rotation. Similarly, Cooper and Shepard (1973) found that RT to judge rotated alphanumeric characters to be normal or backward increased with their angular departure from the upright, and inferred that their subjects mentally rotated each character to the upright position before making the judgment.

Shepard and Judd (1976) also showed that mental rotation has properties in common with apparent rotation. They presented pictures of three-dimensional figures in alternation at two orientations to produce an illusion of rigid rotation, and found that the minimum cycle duration at which the illusion broke down increased linearly with the angular distance between the orientations and at the same slope for rotations in depth and in the plane of the picture. These properties paralleled those of mental rotation of the same objects, as reported by Shepard and Metzler (1971), except that the inferred rate of mental rotation was considerably slower than the maximum rate

This experiment was carried out as part of an MSc project by the first author. The idea for the experiment arose out of discussions with Pierre Jolicoeur. The authors wish to thank Jennifer Freyd, Pierre Jolicoeur, and an anonymous reviewer for their helpful comments on an earlier draft of this paper. Correspondence should be addressed to P. M. Corballis, Department of Psychology, Columbia University, Schermerhorn Hall, New York, NY 10027. of perceived apparent rotation. Shepard and Judd argued that the two depend on the same imaginal mechanisms, but that imagery generally proceeds more rapidly when driven externally than when generated internally. These findings suggest that mental rotation, like physical rotation, is a smooth, analog process rather than a propositional one (see also Shepard \& Cooper, 1982). In further support of this, Cooper (1976) has reported evidence that when subjects mentally rotate a shape between two orientations, the shape is coherently represented at intermediate orientations along the rotation trajectory. If rotation were accomplished in some propositional or symbolic manner (as on a digital computer, for example), one would not generally expect representations at intermediate orientations to be computed.

On the other hand, as Anderson (1978) has pointed out, one can mimic analog rotation by using a propositional model. Palmer (1975), for example, has proposed a model in which locations of points on a figure are represented in polar coordinates, and rotation is accomplished by changing the coordinate representing angular orientation in small, successive increments. By choosing increments that are small enough, such a model can produce, to any desired level of approximation, a linear function relating rotation time to angle of rotation, and it can also account for the intermediate representations inferred by Cooper (1976). This may seem to be a post hoc way of preserving a propositional account, and successive transformations through small steps may seem too laborious to be computationally plausible (Kosslyn \& Pomerantz, 1977; Wilton, 1978). Nevertheless, Morgan (1983) has argued that if spatial relations are represented symbolically but nonmetrically, then it may indeed be plausible to suppose that spatial transformations are accomplished through the 
iterative or recursive application of minimal adjustments. $\mathrm{He}$ cites the evidence of multiple eye movements during mental rotation, reported by Carpenter and Just (1978), in support of such a model. However, Carpenter and Just's data can also be taken to imply partwise rotation of complex figures, with each part nevertheless rotated in analog fashion (Shepard \& Cooper, 1982).

Instead of arguing from the properties of mental rotation, as Shepard and his colleagues have done, an alternative approach has been to seek direct interactions between mental rotation and perceived rotation in order to determine what they have in common. For example, Corballis (1986) and Corballis and McLaren (1982) had subjects watch a rotating, textured disk prior to the presentation of letters that were to be mentally rotated to the upright position. This induced a rotation aftereffect, causing the letters to be perceived as though rotating in the opposite direction, although paradoxically they did not appear to change their actual orientations. This aftereffect did influence subsequent mental rotation of the letters, suggesting common mechanisms. Specifically, mental rotation appeared to be slowed by an aftereffect in the direction opposite to the shortest angular route to the upright position, but not to be speeded by an aftereffect in the same direction as the shortest angular route (Corballis, 1986). There remains some doubt, however, regarding the level at which this slowing occurred. One possibility is that the actual rate of rotation was decreased. It is perhaps more likely, though, that the aftereffect may have sometimes induced subjects to rotate "the long way around." For example, a letter tilted $120^{\circ}$ from the upright position might sometimes have been rotated through the larger angle of $240^{\circ}$, if the aftereffect was in that direction.

Jolicoeur and Cavanagh (1992) showed that the rate of mental rotation was not affected if the stimuli to be rotated were presented against a rotating background, suggesting that any mechanisms in common between mental rotation and perceived rotation must be located beyond the level of motion-sensitive cells. Rotation performance was also very similar, whether the stimuli were defined by luminance, color, texture, motion, or stereoscopic depth, further suggesting that the mental-rotation operator is beyond the early stages of visual analysis. However, they did find that mental rotation was influenced by apparent motion of the stimulus. Each stimulus was presented at two orientations, $10^{\circ}$ apart in quick succession, so that it appeared to rotate. Responses on the mental-rotation task were faster when the apparent rotation was in the direction of the postulated mental rotation than when it was in the opposite direction. Jolicoeur and Cavanagh inferred that there must indeed be some relatively high level of processing at which mental rotation and the perception of apparent motion share common mechanisms. However, they were unable to rule out the possibility that the apparent motion sometimes caused subjects to mentally rotate the stimulus through the larger angle, as proposed by Corballis and McLaren (1982).
Corballis and Blackman (1990) have also investigated the effect of apparent movement on mental rotation, and suggested yet another mechanism for the interaction between the two. They presented subjects with a single hand on a clockface, which appeared in two consecutive positions $30^{\circ}$ apart (e.g., at 3:00, then at 4:00) so that it appeared to move from one to the other. The task was to decide whether a small bar perpendicular to one side of the clockhand would point to the right or to the left if it was in the upright (12:00) position, a task that evidently required mental rotation of the stimulus to the upright position. The function relating RT to the orientation of the jump suggested that the jump influenced the perceived orientation from which the clockhand was mentally rotated, and in some cases caused it to be extrapolated beyond the end of the jump. That is, the jump caused the function to be shifted along the orientation axis.

Corballis and Blackman (1990) suggested that this extrapolation may have been due to representational momentum (Freyd \& Finke, 1985), in which the final orientation of a shape subjected to apparent rotation is remembered as though it was extrapolated beyond that orientation. However, this explanation requires qualification, at least. In the majority of cases (13 out of 22), the estimated orientation from which mental rotation began was within the jump rather than extrapolated beyond it and, when it was extrapolated, the degree of extrapolation did not conform well to estimates derived from studies of representational momentum. Freyd and Finke found that the relation between the implied velocity $(\nu)$ of an apparent rotation and the extent $(\theta)$ of the extrapolation could be approximated by the following equation:

$$
\theta=1.90+0.019 \nu .
$$

Applying this equation to the angular velocity employed by Corballis and Blackman $\left(214^{\circ} / \mathrm{sec}\right)$ yields an expected extrapolation of about $6^{\circ}$, whereas the estimated extrapolations actually ranged from $5^{\circ}$ to $44^{\circ}$, with a mean of $18.6^{\circ}$.

Freyd and Johnson (1987) also found that the extrapolation increased linearly with the interval between final presentation of the stimulus itself and the test probe; that is, it was a function of memory rather than of perception. If the interval was only $50 \mathrm{msec}$, for example, the extrapolation was only about $1^{\circ}$. Consequently, if we assume that the subjects in Corballis and Blackman's experiment began mental rotation very soon after the second presentation of the clockhand, the predicted adjustment to the mental-rotation function would be much too small to have been measurable. For similar reasons, Jolicoeur and Cavanagh (1992) also ruled out representational momentum as an explanation for the effect of apparent rotation on mental rotation in their experiment.

There is an important difference, though, between the representational momentum paradigm and the mentalrotation tasks used by Corballis and Blackman (1990) and by Jolicoeur and Cavanagh (1992). In the representational momentum paradigm, subjects were asked to make judg- 
ments as to the actual final orientation of a shape that had been subjected to apparent rotation, whereas in the mentalrotation experiments, they were not constrained to rotate from either of the two actual orientations that defined a jump. The subjects may have interpolated the clockhand within the jump and extrapolated it beyond the jump, and any explanation may have been beyond the limits of representational momentum, as defined by Freyd and her colleagues. In the context of mental-rotation experiments, then, extrapolation might be better attributed to overshoot than to representational momentum.

If the subjects sometimes rotated from an interpolated rather than an extrapolated orientation, another issue arises. Just as there has been some question as to whether intermediate orientations are computed in mental rotation, so has there been doubt as to whether they are computed in apparent motion. Kolers (1964) found that real movement of a stimulus masked the detection of a target in its path, whereas apparent motion did not. This raises some question as to the perceptual reality of a stimulus in the locations intermediately between those of its actual presentations, suggesting that perhaps the impression of smooth movement may be essentially an unconscious inference rather than a fact of neural computation. This suggests that the interpolation inferred by Corballis and Blackman (1990) may not have been a genuine interpolation, but may have resulted from the averaging of functions based on the two actual presentations.

The main purpose of the present experiment was to further study the effect of apparent rotation on mental rotation, while removing the ambiguity arising from the two presentations of the stimulus. The experiment was essentially a replication of Corballis and Blackman's (1990) study, except that the bar was presented on just one of the two presentations of the clockhand. This created the perceptual impression of apparent motion, yet allowed the orientation of the critical stimulus itself to be specified uniquely. On half of the trials the bar appeared only on the first presentation, and on the other half it appeared only on the second presentation.

This procedure also allowed us to compare the relative contributions of each presentation of the clockhand with any effect of apparent rotation. If the bar appears on the second presentation of the clockhand, but the estimated orientation from which mental rotation begins is extrapolated beyond the second presentation, then we might indeed infer a process of overshoot, and it would remain to be seen whether the extent of this effect would conform to estimates of representational momentum, as derived from the studies by Freyd and her colleagues. However, if there is an effect when the bar appears on the first presentation, then this might be attributed to representational drag, in which the second presentation of the clockhand "drags" the perceived orientation of the stimulus along its path. Put more simply, would the second presentation be pushed by the preceding motion, or would the first be pulled by the following motion?

\section{METHOD}

\section{Subjects}

The subjects were 11 adult volunteers, 6 men and 5 women, ranging in age from 19 to 30 years. All had normal or corrected-tonormal vision.

\section{Apparatus and Stimuli}

The stimuli were generated by an Apple IIe microcomputer. They were presented in amber with a rapid-decay phosphor on a dark screen, and were synchronized with the $50-\mathrm{Hz}$ frame rate (Cavanagh \& Anstis, 1980). They were presented against a clockface represented by 12 dots arranged in a circle at $30^{\circ}$ intervals (i.e., corresponding to the positions of the hours), with a radius of $30 \mathrm{~mm}$, so that it subtended a visual angle of $3^{\circ}$ when viewed from a distance of $57 \mathrm{~cm}$. Each clockhand consisted of a line drawn from the center of the circle to one of the dots, followed by a similar line, displaced in either direction by $30^{\circ}$. In addition, a small bar subtending $.5^{\circ}$ of visual angle appeared halfway along one of the clockhands, and perpendicular to it. This bar could appear on either side of the clockhand. On half of the trials it appeared on the first presentation of the clockhand, and on the other half it appeared on the second presentation.

Each clockhand appeared for seven frames, or $140 \mathrm{msec}$, with no intervening interval, resulting in a clear impression of apparent rotation, as though a single line moved from one orientation to the other at an implied angular velocity of $214^{\circ} / \mathrm{sec}$. The screen then went blank.

There were 96 different stimuli: The clockhand could sweep out 12 different $30^{\circ}$ sectors; each jump was either clockwise or counterclockwise; for each jump the bar was on either the right or left of the clockhand relative to its upright orientation; and the bar could appear on either the first or second presentation of the clockhand. In a given experimental session, each stimulus was presented twice, for a total of 192 trials that were randomly ordered.

\section{The Task}

The subjects were required to press the " $N$ " key with the right index finger if the bar would be on the right of the clockhand if it were in the upright $(12: 00)$ position, and the " $B$ " key with the left index finger if it would be on the left. These keys are adjacent in the center of the bottom row of the keyboard. The subjects were instructed to respond as quickly and accurately as possible. The RTs were measured from the offset of the presentation of the clockhand with the bar.

\section{Procedure}

Each subject participated in a single session of 192 trials, preceded by 10 practice trials in which stimuli were chosen at random from the 96 possible stimuli. Viewing distance was maintained at approximately $57 \mathrm{~cm}$ by placing the subject's chin on a chinrest. Before each trial, the subject sat with the index finger of each hand resting lightly over the appropriate response keys. The subject initiated the trial by pressing the space bar, and the stimulus appeared $500 \mathrm{msec}$ later. If the subject made an error, that trial was repeated at the end of the session.

\section{RESULTS}

\section{Reaction Times}

The RTs for correct responses were subjected to an analysis of variance (ANOVA), in which the independent variables were orientation, direction of the jump (clockwise vs. counterclockwise), side of the clockhand on 
which the bar appeared (left vs. right, relative to the 12:00 position), and presentation of the clockhand on which the bar appeared (first vs. second). $F$ ratios for repeated measures effects involving 2 or more degrees of freedom were assessed with both regular and conservative degrees of freedom (Winer, 1971). When these were discrepant, degrees of freedom were reduced by the multiplier epsilon, estimated according to the procedures described by Greenhouse and Geisser (1959). Only the $F$ ratios that met the .05 level or better, according to the reduced degrees of freedom, were accepted as significant.

There was a highly significant main effect of orientation, $\left[F(11,110)=21.11, M S_{\mathrm{e}}=173,643, p<.001\right]$ as well as significant interactions between orientation and direction of the jump $\left[F(11,110)=3.13, M S_{e}=76,694\right.$, $p<.001]$, orientation and side of the bar $[F(11,110)=$ $\left.6.29, M S_{\mathrm{c}}=137,714, p<.001\right]$, and orientation, direction of the jump, and presentation on which the bar appeared $\left[F(11,110)=2.88, M S_{\mathrm{e}}=80,398, p<.01\right]$.

Mean RTs for each orientation and side of the bar are plotted in Figure 1. This figure shows that the side of the bar influenced the direction of mental rotation, since the function for the bar on the right of the clockhand peaks at $150^{\circ}$ and is skewed to the left, whereas the function for the bar on the left peaks at $180^{\circ}$ and is skewed to the right. These peak shifts are not accompanied by corresponding shifts in the troughs; indeed, the minimum RT in the case of the bar to the right is shifted in the opposite direction. This pattern of results is not consistent with a simple shift along the abscissa, as one would expect if the side of the bar influenced perceived orientation, but rather suggests that mental rotation itself was biased in the direction of the bar. In other words, there may have been a tendency to rotate in the direction toward which the bar points, even though this sometimes meant rotating "the long way around." This effect did not interact in any way with direction of the jump.

The interaction between orientation, direction of the jump, and presentation of the clockhand on which the bar appeared is plotted in Figure 2. It can be seen that when

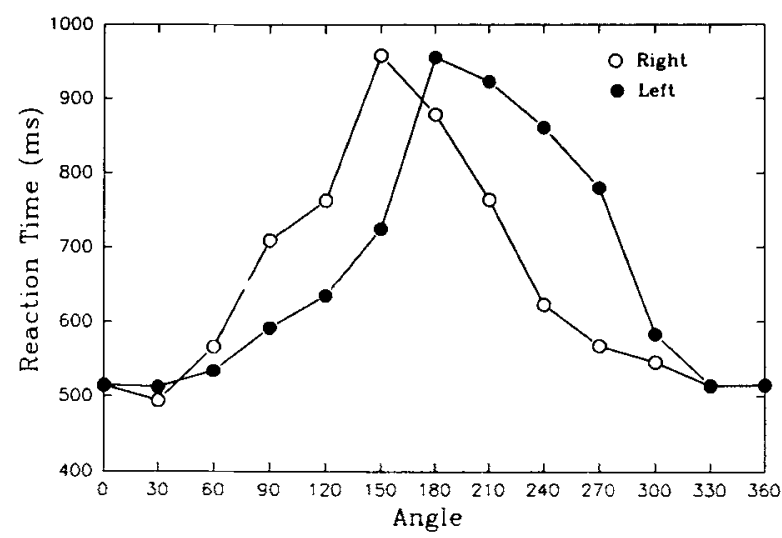

Figure 1. Reaction time as a function of angular orientation for the bar on the left and right sides of the clockface.
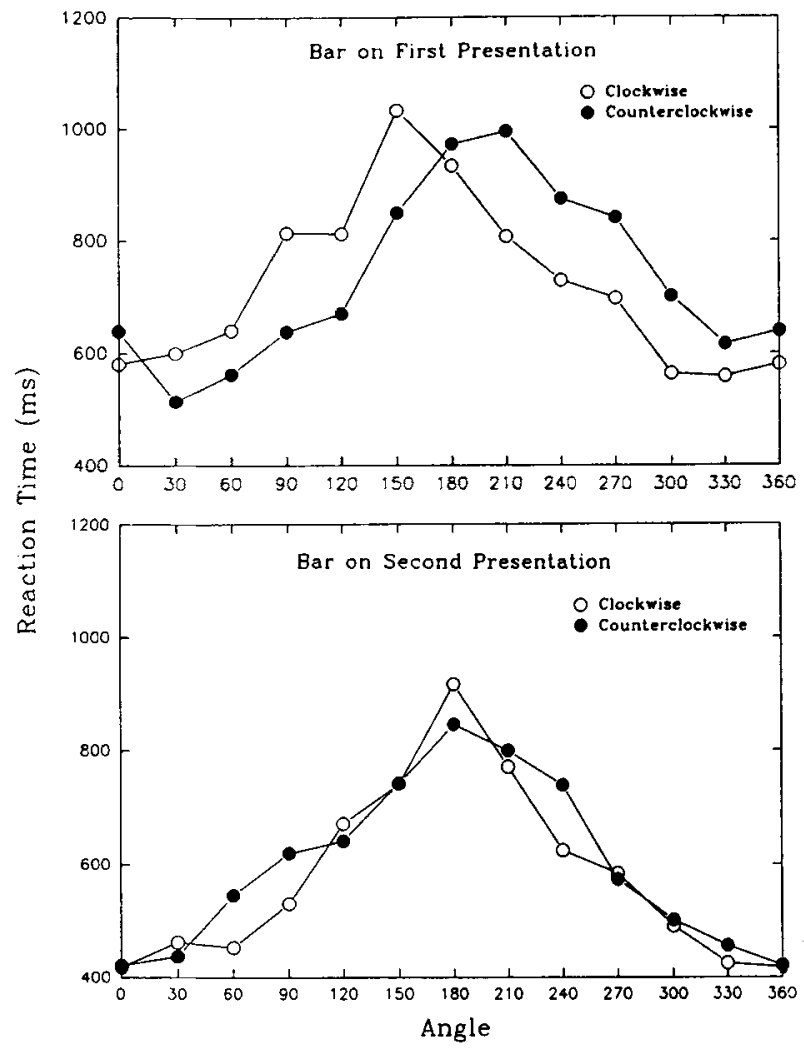

Figure 2. Reaction time as a function of angular orientation for each presentation of the clockhand with the bar and each direction of the jump.

the bar is on the first presentation of the clockhand the function for clockwise jumps peaks at $150^{\circ}$, with a minimum between $300^{\circ}$ and $330^{\circ}$. The function for counterclockwise jumps peaks at $210^{\circ}$, with a minimum at $30^{\circ}$. When the bar is on the second presentation of the clockhand, both functions peak at $180^{\circ}$, with minima at $0^{\circ}$. Thus, mental rotation was affected by apparent rotation only when the bar was on the first presentation of the clockhand.

To investigate this further, the angular adjustment to each orientation that would maximize the fit of the obtained RTs to those predicted by the "idealized" mentalrotation function was calculated for each direction of movement and for each presentation of the clockhand on which the bar appeared. Computational procedures are detailed in the Appendix.

When the bar appeared on the first presentation, the fit for clockwise jumps was maximized when the adjustment was $21^{\circ}$ clockwise from each orientation. This can be interpreted to mean that the representation of the stimulus was "dragged" to a position $21^{\circ}$ clockwise from where it actually appeared, before being mentally rotated to the upright position. This fit accounted for $93.21 \%$ of the variance. The estimated rate of mental rotation, given by the slope of the mental-rotation function, was $374^{\circ} / \mathrm{sec}$, 
which is well within the range typically found in mentalrotation experiments (e.g., Cooper \& Shepard, 1973). Similarly, the fit for counterclockwise jumps, when the bar was on the first presentation, was maximized when the adjustment was $28^{\circ}$ counterclockwise, implying a drag of $28^{\circ}$. This fit accounted for $95.62 \%$ of the variance and yielded a rate of mental rotation of $377^{\circ}$ per second.

When the bar was on the second presentation, the fit for clockwise jumps was maximized when the adjustment was $3^{\circ}$ clockwise, which accounted for $92.32 \%$ of the variance and yielded a rate of mental rotation of $385^{\circ} / \mathrm{sec}$. The fit for counterclockwise jumps was maximized when the adjustment was $5^{\circ}$ clockwise, which accounted for $88.05 \%$ of the variance and yielded an estimate of the mental-rotation rate of $395^{\circ} / \mathrm{sec}$. These adjustments are not consistent with the value of about $6^{\circ}$ in the direction of the jump that would be expected if representational momentum is distorting the perceived location of the stimulus (Freyd \& Finke, 1985), and suggest, in fact, that there is little or no effect on mental rotation when the bar appears on the second presentation.

Angular adjustments of the mental-rotation functions were also calculated for each subject; the resulting parameters are summarized in Table 1 . They are reasonably compatible with mental rotation in practically every case, with the estimated rates of rotation nearly all falling within the range of $164^{\circ}$ to $800^{\circ} / \mathrm{sec}$, as reported by Cooper and Shepard (1973). When the bar was on the first presentation, the adjustments are similar to those reported by Corballis and Blackman (1990), although they

Table 1

Estimated Parameters Obtained by Fitting Idealized Mental-Rotation Functions to RTs for Each Subject

\begin{tabular}{|c|c|c|c|c|c|c|}
\hline \multirow[b]{2}{*}{ Subject } & \multicolumn{3}{|c|}{ Clockwise Jumps } & \multicolumn{3}{|c|}{ Counterclockwise Jumps } \\
\hline & Adjustment* & VAF & Rate & Adjustment* & VAF & Rate \\
\hline \multicolumn{7}{|c|}{ Bar on First Presentation of Clockhand } \\
\hline $\begin{array}{r}1 \\
2 \\
3 \\
4 \\
5 \\
6 \\
7 \\
8 \\
9 \\
10 \\
11\end{array}$ & $\begin{array}{r}-6.3 \\
+27.6 \\
+30.0 \\
-9.0 \\
-10.9\end{array}$ & $\begin{array}{l}37.9 \\
70.1 \\
43.9 \\
22.9 \\
33.1 \\
83.4 \\
51.7 \\
85.9 \\
83.6 \\
64.0 \\
47.4\end{array}$ & $\begin{array}{r}514 \\
459 \\
452 \\
1037 \\
336 \\
118 \\
557 \\
283 \\
396 \\
317 \\
702\end{array}$ & $\begin{array}{r}-24.3 \\
+23.2 \\
-32.7 \\
-36.0 \\
-35.1 \\
-23.8 \\
-40.7 \\
-8.5 \\
-47.6 \\
-52.5 \\
-28.5\end{array}$ & $\begin{array}{l}73.4 \\
39.4 \\
61.1 \\
45.3 \\
67.1 \\
77.5 \\
65.3 \\
87.6 \\
59.1 \\
66.4 \\
75.3\end{array}$ & $\begin{array}{l}334 \\
388 \\
435 \\
671 \\
199 \\
144 \\
444 \\
266 \\
352 \\
438 \\
584\end{array}$ \\
\hline \multicolumn{7}{|c|}{ Bar on Second Presentation of Clockhand } \\
\hline 1 & -13.9 & 60.2 & 377 & +50.0 & 33.9 & 527 \\
\hline 2 & +10 & 71.7 & 319 & -13.4 & 65.6 & 355 \\
\hline 3 & -12.5 & 73.0 & 360 & -11.9 & 58.3 & 358 \\
\hline 4 & -3.3 & 54.5 & 522 & +23.3 & 58.6 & 541 \\
\hline 5 & 0.0 & 48.0 & 257 & -13.1 & 68.2 & 257 \\
\hline 6 & +16.0 & 89.8 & 147 & +9.9 & 77.3 & 163 \\
\hline 7 & -13.7 & 75. & 351 & -9.1 & 32.6 & 600 \\
\hline 8 & -8.6 & 72.4 & 514 & -17.0 & 66.3 & 409 \\
\hline 9 & -3.5 & 38.9 & 825 & -23.6 & 73.3 & 352 \\
\hline 10 & -13.2 & 61.5 & 340 & -17.3 & 48.1 & 529 \\
\hline 11 & -6.3 & 53.6 & 653 & -6.3 & 59.7 & 406 \\
\hline
\end{tabular}

Note-VAF $=$ percent variance accounted for. ${ }^{*}$ In degrees. are slightly more variable; for 16 of the 22 functions, the adjustment was in the direction of the apparent rotation, compared with 21 out of 22 in Corballis and Blackman's experiment. When the bar was on the second presentation, only 10 of the 22 functions were influenced in the direction of the apparent rotation, with one showing no influence.

An ANOVA of the angular adjustments revealed a significant interaction between direction of the jump and presentation on which the bar appeared $[F(1,10)=18.48$, $\left.M S_{\mathrm{e}}=2881, p<.01\right]$. When the bar appeared on the first presentation of the clockhand the mean adjustment for clockwise jumps was $14.1^{\circ}$ clockwise, whereas the mean adjustment for counterclockwise jumps was $28^{\circ}$ counterclockwise. When the bar was on the second presentation of the clockhand, the corresponding values were $4.5^{\circ}$ counterclockwise and $2.5^{\circ}$ counterclockwise. Tests of simple effects (e.g., Winer, 1971) showed that when the bar appeared on the first presentation of the clockhand, the effect of direction was highly significant $[t(10)=$ $4.97, p<.001]$, but when it appeared on the second presentation of the clockhand, there was no significant effect of direction $[t(10)=0.25$, n.s.].

Further tests were carried out to determine whether the adjustments for each direction of the jump and presentation on which the bar appeared were significantly greater than zero. When the bar appeared on the first presentation, the adjustments for both clockwise jumps $[t(10)=$ $2.05, p<.05]$ and counterclockwise jumps $[t(10)=$ $3.81, p<.01]$ were significantly different from zero, but when it appeared on the second presentation, neither adjustment differed significantly from zero $[t(10)=1.47$ and 0.39 , respectively, n.s.]. The adjustments for each subject (see Table 1) show no sign of a bimodal distribution, with peaks at $0^{\circ}$ and $30^{\circ}$, as might be expected if the mental rotation was carried out from one of the two positions of the clockhand.

To check that the effect of the jump was best explained in terms of a shift in the perceived orientation of the stimulus, the orientations of the stimuli were relabeled such that the orientation closest to the minimum of the mentalrotation function corresponded to the upright position. Thus, the orientation of the stimulus that was perceived as closest to the upright position was labeled $0^{\circ}$, the next was labeled $30^{\circ}$, and so on. For example, for Subject 7, when the bar was on the first presentation of the clockhand, the adjustment for clockwise jumps was $35^{\circ}$ clockwise. This may also be interpreted to mean that for clockwise jumps beginning at the upright (12:00) position, the bar was mentally rotated from about $5^{\circ}$ beyond the $30^{\circ}$ position (1:00). Thus, the $30^{\circ}$ position was relabeled $0^{\circ}, 60^{\circ}$ was relabeled $30^{\circ}$, and so forth. These adjustments had the effect of maximizing the fits to an idealized mental-rotation function, in which RT rises linearly from $0^{\circ}$ to $180^{\circ}$, and then falls linearly from $180^{\circ}$ to $360^{\circ}$. The subjects' RT functions were therefore effectively aligned to within $15^{\circ}$.

An ANOVA of the adjusted RTs indicated that the adjustments preserved the significant effect of orientation 
$\left[F(11,110)=23.92, M S_{\mathrm{e}}=45,506, p<.001\right]$, but removed the significant interactions between orientation and direction of the jump $\left[F(11,110)=1.18, M S_{c}=16,340\right.$, n.s.] as well as between orientation, direction of the jump, and presentation of the clockhand on which the bar appeared $\left[F(11,110)=.71, M S_{e}=12,853\right.$, n.s. $]$. Mean RTs for each direction of jump and each presentation of the clockhand on which the bar appeared are plotted as a function of the relabeled orientations in Figure 3. Note that the functions are effectively superimposed, with no systematic deviation from a typical mental-rotation function. This suggests that the original distortions were indeed caused by changes in the perceived location from which the stimuli were rotated, rather than by influencing the rate of mental rotation.

\section{Errors}

The subjects made errors on $5.3 \%$ of the trials. The errors were subjected to an ANOVA, with the same independent variables as in the RT analysis. There was a significant main effect of orientation $[F(11,110)=5.33$, $M S_{\mathrm{e}}=0.217, p<.0011$, with errors at a minimum at $330^{\circ}(1.4 \%)$, rising to a maximum at $210^{\circ}(12.9 \%)$. The interaction between orientation and side of the bar did not approach significance $\left[F(11,110)=1.39, M S_{c}=0.137\right.$, n.s.]. The error rates for this interaction are shown in Table 2.

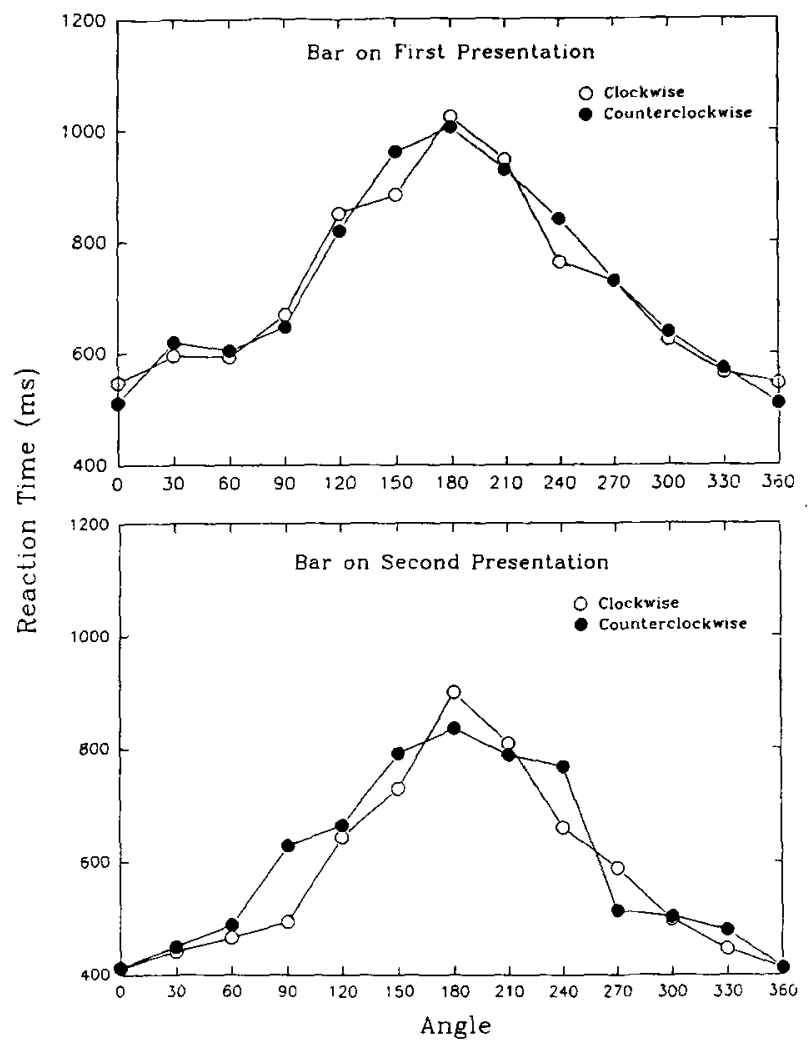

Figure 3. Reaction time as a function of adjusted orientation for each presentation of the clockhand with the bar and each direction of the jump.
Table 2

Mean Error Rates as a Percentage of Trials at Each Orientation for Each Side of the Clockhand on Which the Bar was Presented

\begin{tabular}{crr}
\hline \multirow{2}{*}{$\begin{array}{c}\text { Orientation } \\
\text { (in Degrees) }\end{array}$} & \multicolumn{2}{c}{ Bar Presentation } \\
\cline { 2 - 3 } & Left & Right \\
\hline 0 & 2.2 & 1.1 \\
30 & 2.8 & 2.2 \\
60 & 2.8 & 0.6 \\
90 & 3.3 & 4.9 \\
120 & 4.3 & 10.2 \\
150 & 7.4 & 9.7 \\
180 & 14.1 & 11.6 \\
210 & 9.3 & 5.9 \\
240 & 6.4 & 7.4 \\
270 & 2.8 & 7.4 \\
300 & 3.3 & 2.2 \\
330 & 1.7 & 1.1 \\
\hline
\end{tabular}

There was a significant interaction between orientation, direction of the jump, and presentation of the clockhand on which the bar appeared $\left[F(11,110)=2.59, M S_{c}=\right.$ $0.0695, p<.01]$. This interaction showed a similar trend to the corresponding interaction in the RT analysis, and was contrary to the predictions of a speed-accuracy tradeoff. The error rates for this interaction are shown in Table 3.

\section{DISCUSSION}

In this experiment, the "mental-rotation" function relating RT to the angular orientation of a stimulus was distorted in two independent ways. First, it was influenced by the direction of the bar that constituted the critical feature of the stimulus. Specifically, the subjects may have been induced on at least some of the trials to rotate in the direction of the bar, even if this meant rotating more than $180^{\circ}$; this would explain why the peak RT was at $150^{\circ}$ when the bar pointed right, and at $210^{\circ}$ when it pointed left. This effect was similar to that induced by a rotation aftereffect (Corballis, 1986; Corballis \& McLaren, 1982). Since the direction of the bar itself was not correlated with the direction of the induced apparent motion, it might be regarded as dynamic information implicit in the stimulus itself. Freyd (1987) has argued that dynamic information of this sort may play a general role in perception, even in the absence of observed real-time change.

Second, the mental-rotation function was influenced by the direction of the jump, but only when the bar appeared on the first presentation. Since there was little evidence that the function was systematically distorted when the bar appeared on the second presentation, representational momentum appears to have had little measurable influence on mental rotation. As we pointed out in the introduction, however, the predicted effect of representational momentum, according to the work of Freyd and Finke (1985) and of Freyd and Johnson (1987), is very small and probably negligible in terms of precision with which any extrapolation could be estimated from mental-rotation functions. 
Table 3

Mean Error Rates as a Percentage of Trials at Each Orientation for Each Direction of Apparent Rotation and Each Presentation of the Clockhand

Orientation Bar on First Presentation Bar on Second Presentation (in Degrees) CW Jumps CCW Jumps CW Jumps CCW Jumps

\begin{tabular}{rrrrr}
\hline 0 & 1.1 & 4.3 & 1.1 & 0.0 \\
30 & 4.3 & 1.1 & 3.3 & 1.1 \\
60 & 3.3 & 2.2 & 1.1 & 0.0 \\
90 & 3.3 & 4.3 & 3.3 & 5.4 \\
120 & 9.3 & 7.4 & 2.2 & 10.2 \\
150 & 13.7 & 4.3 & 5.4 & 10.2 \\
180 & 10.2 & 20.0 & 10.2 & 10.2 \\
210 & 4.3 & 12.0 & 6.4 & 7.4 \\
240 & 2.2 & 12.9 & 5.4 & 6.4 \\
270 & 2.2 & 9.3 & 5.4 & 3.3 \\
300 & 1.1 & 4.3 & 2.2 & 3.3 \\
330 & 1.1 & 2.2 & 2.2 & 0.0 \\
\hline
\end{tabular}

Note-CW = clockwise CCW $=$ counterclockwise.

What, then, might explain the effect of apparent motion when the bar appeared on the first presentation of the clockhand? On a priori grounds, there are perhaps three ways in which processing of the first presentation might have been influenced by the second:

1. The second presentation might simply have delayed, or interrupted, processing of the first. This does not explain why the effect varied with angular orientation-why the peaks and troughs of the function relating RT to angular orientation were shifted in the direction of the apparent rotation.

2 . The apparent motion induced by the second presentation might have guided the path of mental rotation itself-what one might term path-guided mental rotation by analogy with what Shepard and Zare (1983) have termed path-guided apparent motion. In the extreme case, the direction of mental rotation would always be in the direction of the apparent motion, giving rise to a linear (rather than a bilinear) RT function for each direction of apparent motion. Clearly, this was not the case. However, it might be argued that the apparent rotation influenced the path of mental rotation only when there was a relatively large angular distance to the upright position. For example, if the shortest distance to the upright position was $150^{\circ}$ clockwise, counterclockwise apparent motion might induce a subject to rotate through $210^{\circ}$ counterclockwise.

If this were the case, the effect should resemble that of the direction of the bar itself on the RT function; that is, it should influence the peak rather than the trough of the function. Since the function tends to flatten in the vicinity of the upright orientation (Cooper \& Shepard, 1973), shifts in the trough are less easily detected than shifts in the peak. Even so, Figure 2 suggests shifts in both peaks and trough, in contrast to the distortions evident in Figure 1 . The results strongly suggest that the effect of the second presentation was simply to shift the functions along the abscissa, rather than to distort along the ordinate.
3. The apparent motion might cause a subject to rotate the first stimulus from some orientation that is displaced in the direction of the second presentation. That is, the second presentation might "drag" the first stimulus some distance along its path, and cause the subject to mentally rotate from this new orientation rather than from the actual orientation. Such an explanation is indeed consistent with outcomes of the analyses.

The results of Corballis and Blackman's (1990) experiment can also very largely be attributed to drag rather than momentum, since they were very similar to those obtained here when the bar was on the first presentation. The effect was, if anything, slightly stronger and more consistent in their experiment, however, perhaps because the appearance of the bar on both presentations of the clockhand produced a slightly stronger perception of movement.

Even when the bar was present on the first presentation, the adjustment was greater than $30^{\circ}$ for 9 of the 22 functions, as it was for 10 of the 22 functions in Corballis and Blackman's (1990) experiment. This suggests that the notion of representational drag should not be thought of as incompatible with that of representational momentum; that is, a stimulus that is "dragged" may still have sufficient "momentum" to carry it beyond the endpoint of the jump. Even in these cases, however, the extrapolations were much more variable than the effects of representational momentum reported by Freyd and Finke (1985) and, in some cases, much larger. As we explained in the introduction, the term overshoot may be more apt, since it implies greater flexibility than representational momentum, as studied by Freyd and her colleagues.

It is in one respect surprising that the "pull" of the second presentation should have influenced the mentalrotation function, whereas the "push" from the first did not. In the first case, the bar appeared on the first presentation and there was consequently no need to attend to the second presentation, so one might have expected the second presentation to have had little effect. But in the second case, the subjects were compelled to attend to both presentations, and one might have expected the impression of motion to be stronger. Presumably, the two presentations were too rapid to allow the subjects to disengage attention between them.

However, there is another respect in which it is not surprising that the influence was one of pull rather than push, since the predominant impression of motion is between the two presentations rather than beyond them, notwithstanding the evidence for overshoot in some cases. Furthermore, the effect of pull, or drag, was such that the subjects typically rotated from some perceived location of the clockhand that did not match either of the actual locations, adding to the evidence of Corballis and Blackman (1990) that there is a "perceptual reality" to the illusory locations in the path of the apparent movement, notwithstanding the negative evidence of Kolers (1964). The illusory representation is presumably not simply a 
result of the statistical averaging of functions based on the two actual orientations, since the stimulus itself appeared at only one of the orientations.

If our interpretation of results is correct, this illusory representation may serve as the starting point for a subsequent mental rotation. This supports the idea that there is a continuity of representation in both apparent motion and mental rotation, and that the same representations may be involved in each (cf. Shepard \& Judd, 1976). However, the act of mental rotation itself clearly seems to be distinct from that of apparent motion, since on half of the trials the two proceeded in opposite directions; that is, the stimulus was first dragged in one direction by the apparent rotation, and then was mentally rotated in the opposite direction back to the upright position. Hence, although apparent rotation and mental rotation may involve the same intermediate representations, there is still no compelling evidence that the one directly affects the other. The most parsimonious interpretation of the results to date is that perceived motion may alter the orientation from which mental rotation takes place (Corballis \& Blackman, 1990; the present results), or may alter the direction of mental rotation (Corballis, 1986; Corballis \& McLaren, 1982).

\section{REFERENCES}

ANDERSON, J. R. (1978). Arguments concerning representations for mental imagery. Psychological Review, 85, 249-277.

Carpenter, P. A., JUST, M. A. (1978). Eye fixations during mental rotation. In J. W. Senders, D. F. Fisher, \& R. A. Monty (Eds.), Eye movements and the psychological functions (pp. 115-133). Hillsdale, NJ: Erlbaum.

Cavanagh, P., Anstis, S. M. (1980). Visual psychophysics on the Apple II: Getting started. Behavior Research Methods \& Instrumen tation, 12, 614-626.

COOPER, L. A. (1976). Demonstration of a mental analog of an external rotation. Perception \& Psychophysics, 19, 296-302.

CoOper, L. A., \& Shepard, R. N. (1973). Chronometric studies of the rotation of mental images. In W. G. Chase (Ed.), Visual information processing (pp. 75-176). New York: Academic Press

Corbaluis, M. C. (1986). On imagined revolutions. In D. F. Marks (Ed.), Theories of image formation (pp. 151-168). New York: Brandon House.

Corballis, M. C., \& Blackman, A. R. (1990). The effect of apparent movement on mental rotation. Memory \& Cognition, 18, 551-555.

Corballis, M. C., MCLAREN, R. (1982). Interaction between perceived and imagined rotation. Journal of Experimental Psychology: Human Perception \& Performance, 8, 215-224.

FREYD, J. J. (1987). Dynamic mental representations. Psychological Review, 94, 427-438.

FreYD, J. J., \& FinKe, R. A. (1985). A velocity effect for representational momentum. Bulletin of the Psychonomic Society, 23, 443-446.

FREYD, J. J., \&OHNSON, J. Q. (1987). Probing the time course of representational momentum. Journal of Experimental Psychology: Learning, Memory, \& Cognition, 13, 259-268.

Greenhouse, S. W., Geisser, S. (1959). On methods in the analysis of profile data. Psychometrika, 24, 95-112.

Jolicoeur, P., \& Cavanagh, P. (1992). Mental rotation, physical rotation, and surface media. Joumal of Experimental Psychology: $\mathrm{Hu}$ man Perception \& Performance, 18, 371-384.

Kolers, P. A. (1964). The illusion of movement. Scientific American, 211, 98-106.

Kosslyn, S. M., \& Pomerantz, J. R. (1977). Imagery, propositions, and the form of internal representations. Cognitive Psychology, 9, 52-76.
Morgan, M. J. (1983). Mental rotation: A computationally plausible account of transformation through intermediate steps. Perception, 12, 203-211.

Palmer, S. E. (1975). Visual perception and world knowledge: Notes on a model of sensory-cognitive interaction. In D. A. Norman \& D. E. Rumelhart (Eds.), Explorations in cognition (pp. 279-307). San Francisco: Freeman.

Shepard, R. N., CoOper, L. A. (1982). Mental images and their transformations. Cambridge, MA: MIT Press.

ShEPARD, R. N., JUDD, S. A. (1976). Perceptual illusion of rotation of three-dimensional objects. Science, 191, 952-954.

ShePard, R. N., Metzler, J. (1971). Mental rotation of threedimensional objects. Science, 171, 701-703.

ShePARD, R. N., Z ZARE, S. L. (1983). Path-guided apparent motion. Science, 220, 632-634.

WiLton, R. N. (1978). Explaining imaginal inference by operations in a propositional format. Perception, 7, 563-574.

WINER, B. J. (1971). Statistical principles in experimental design (2nd ed.). New York: McGraw-Hill.

\section{APPENDIX}

\section{Computational Procedures for Angular Adjustments to Mental Rotation Functions}

Suppose the clockhand with the bar is perceived $y^{\circ}$ from the orientation at which it appears and is mentally rotated back to the upright position before a decision about the bar is made. The problem is to find $y$ such that the fit to an idealized " mentalrotation" function is maximized.

First, we suppose that $0 \leq y \leq 30$, and measure orientation in degrees clockwise from the upright position. If the clockhand with the bar is actually at $0^{\circ}$, it is perceived at $y^{\circ}$ so that the perceived angular distance back to the upright position is $y^{\circ}$. We can similarly compute the perceived shortest angular distance from the perceived orientation of the clockhand back to the upright position for all of the actual orientations of the clockhand. For the 12 orientations from $0^{\circ}$ through $330^{\circ}$, these angular distances are $y,(30+y),(60+y),(90+y),(120+y)$, $(150+y),(180-y),(210-y),(240-y),(270-y),(300-y)$, and $(330-y)$, respectively. We assume that the time taken to rotate the clockhand back to the upright position is directly proportional to these values.

We can therefore construct a contrast whose coefficients represent these perceived angular distances. For ease of computation, we divide the angular distances by 30 , substituting $x=$ $y / 30$ and replacing the actual orientations by the normalized coefficients $-3,-2,-1,0,1,2,3,2,1,0,-1$, and -2 . We also number the 12 orientations from 1 to 12 (i.e., so that orientation 1 is $0^{\circ}$, orientation 2 is $30^{\circ}$, etc.). Therefore, if $T_{j}$ is the RT corresponding to actual orientation $j$, the contrast representing mental rotation from the corresponding perceived orientation is

$$
\begin{aligned}
C= & (-3+x) T_{1}+(-2+x) T_{2}+(-1+x) T_{3} \\
& +(x) T_{4}+(1+x) T_{5}+(2+x) T_{6} \\
& +(3-x) T_{7}+(2-x) T_{8}+(1-x) T_{9} \\
& +(-x) T_{10}+(-1-x) T_{11}+(-2-x) T_{12} .
\end{aligned}
$$

This may be written as $C=A+B x$, where

$$
\begin{aligned}
A= & -3 T_{1}-2 T_{2}-T_{3}+T_{5}+2 T_{6} \\
& +3 T_{7}+2 T_{8}+T_{9}-T_{11}-2 T_{12},
\end{aligned}
$$

and 


$$
\begin{aligned}
B= & T_{1}+T_{2}+T_{3}+T_{4}+T_{5}+T_{6}-T_{7} \\
& -T_{8}-T_{9}-T_{10}-T_{11}-T_{12} .
\end{aligned}
$$

$A$ is the contrast representing an idealized mental-rotation function in the absence of any discrepancy between actual and perceived orientation, and $B x$ represents the adjustment if there is a discrepancy of $y^{\circ}$.

The proportion of variance attributable to $C$ is given by

$$
V=\frac{C^{2}}{\Sigma c_{j}^{2}},
$$

where the $c_{j}$ are the coefficients of the contrast. Substituting the values of $c_{j}$ from Equation Al above and simplifying yields

$$
V=\frac{C^{2}}{38-12 x+12 x^{2}} \text {. }
$$

To find the value of $x$ that maximizes $V$, we differentiate $V$ with respect to $x$ and set it to 0 . Solving for $x$ gives

$$
x=\frac{3 A+19 B}{6 A+3 B} .
$$

This solution is acceptable only if $x$ lies between 0 and 1 , and if the value of the contrast $C$ is positive (i.e., if the solution yields a maximum rather than a minimum). The value of $x$ is multiplied by 30 to yield the angular adjustment $y$.

Solutions are sought for each cyclic permutation of the values of $T_{j}$. Thus, in the first permutation, $T_{1}$ is replaced by $T_{2}, T_{2}$ by $T_{3}$, and so on. If the computed value of $x$ lies between 0 and 1 , then the solution identifies an adjustment lying between $30^{\circ}$ and $60^{\circ}$ clockwise of the clockhand on which the bar appeared. For example, if $x=0.5$, the angular adjustment would be $45^{\circ}$ $(y=30 x+30)$ clockwise from the clockhand on which the bar appeared. The second permutation would seek a solution between $60^{\circ}$ and $90^{\circ}$ clockwise of the clockhand on which the bar appeared, and so on. Variances are computed for each acceptable solution from Equation $\mathbf{A} 2$.

Variances are also computed for each orientation, in case there is no maximum between orientations. This is done by setting $x$ to 0 in Equation $A 1$, yielding $V=\mathrm{A}^{2} / 38$. $V$ is computed for each cyclical permutation of the $T_{j}$, thus checking each orientation clockwise from the upright (i.e., this checks the position of the hour hand in 1-h steps from 12:00). The adjustment yielding the largest variance is then taken as the final solution.

(Manuscript received February 10, 1992; revision accepted for publication November $4,1992$. 